\title{
Dynamical mass of a quantum vortex in a Josephson junction array
}

\author{
U. Eckern \\ Institut für Physik, Universität Augsburg, D-86135 Augsburg, Germany \\ G. Luciano and A. Tagliacozzo \\ Dipartimento di Scienze Fisiche, Universita' di Napoli, Mostra d'Oltremare Pad. 19, I-80125 Napoli, Italy \\ and Istituto Nazionale di Fisica della Materia (INFM), I-16152 Genova, Italy \\ (Received 4 February 1997; revised manuscript received 27 June 1997)

\begin{abstract}
The real-time response to a small external perturbation of a vortex in a quantum Josephson junction array, with long-range Coulomb interaction between Cooper pairs, is analyzed. While the static damping is zero for vortex velocities below some threshold value $v_{\text {th }}$ (which implies the possibility of ballistic motion), a dynamical friction due to the coupling to the plasma oscillations is always present for frequencies higher than a given threshold $\omega_{\text {th }}$. The latter approaches zero when the velocity increases to $v_{\text {th }}$. However, radiative dissipation of the vortex affects the threshold for ballistic motion. We discuss the conditions under which a mass can be defined for the vortex as a quantum particle.
\end{abstract}

[S0163-1829(97)05942-0]

\section{INTRODUCTION}

A current distribution in form of vortices can be induced in a two-dimensional superconducting Josephson junction array (JJA) by applying a small uniform magnetic field orthogonally to the lattice. A bias current sets the vortex in motion, and, under suitable conditions, it can move ballistically, thus behaving as a quantum particle that is characterized by a mass. ${ }^{1,2}$ We analyze here these conditions, and we find that when a dynamical mass, $M_{\text {dyn }}$ can be defined, it is in general not identical to the thermodynamic mass $M_{v}$ (usually derived from a vortex effective action in imaginary time). ${ }^{3}$ The dynamical mass relates the acceleration of the vortex to a small external perturbating force, and, in particular, it is an increasing function of the vortex velocity.

In a high-quality JJA, where ohmic dissipation is negligibly small, two mechanisms are responsible for dissipation. The first is excitation of quasiparticles; however this effect is frozen out at low temperatures and low vortex velocities. The second, which is relevant to our discussion, is the coupling to the plasma oscillations: a moving vortex can lose kinetic energy by emitting "spin waves", which restricts the ballistic regime. (Here and in the following, we consider the limit where the nearest-neighbor capacitance, $C_{1}$, is much larger than the capacitance to the ground, $C_{0}$, such that the screening length, $\lambda=\sqrt{C_{1} / C_{0}}$, is much larger than the lattice spacing.) It has been shown ${ }^{4,5}$ that the spin-wave damping is strongly nonlinear, and it is active for vortex velocities larger than a certain threshold, $v_{\text {th }}$.

The first estimate of the mass, $M_{\mathrm{ES}}=\pi^{2} \hbar^{2} / 4 E_{C}$, was obtained in Ref. 1 in the so-called classical limit, $E_{J} \gg E_{C}$. Here $E_{J}$ is the Josephson coupling, and $E_{C}=e^{2} / 2 C_{1}$ is the charging energy; all lengths are in units of the lattice spacing. Decreasing the ratio $E_{J} / E_{C}$ (such that $\alpha=\sqrt{E_{C} / 8 E_{J}}$ $\sim 1$ ), the quantization of the charge on the islands has to be taken into account, for example, numerically and analytically within the self-consistent harmonic approximation (SCHA), ${ }^{6}$ or using a low- $\alpha$ expansion. ${ }^{7}$ The low- $\alpha$ expansion yields, in contrast to the SCHA - but physically correct- the EckernSchmid result, $M_{\mathrm{ES}}$, when $\alpha$ goes to zero. The thermody- namic mass, $M_{v}$, which is nothing but the second variation of the imaginary time action with respect to the vortex trajectory, was studied in detail before. In particular, it was noticed ${ }^{6}$ that, by increasing $\alpha$, the vortex mass vanishes at a critical value, which can be taken as the signature of the superconductor-insulator phase transition. This quantum phase transition has been predicted in Ref. 3 and indeed was experimentally observed. ${ }^{8}$ An indicator of the "quantumness" of the vortex motion is the length $\xi$ for charge-charge correlations, which increases with $\alpha$ and diverges at the transition.

In Sec. II we study the equation of motion for the vortex coordinate in real time, which is derived from the Euclidean action using standard analytical continuation (see, for example Ref. 9). The frequency-dependent linear response is analyzed in Sec. III, while in Sec. IV we consider the nonlinear response of the moving vortex when the junctions in its wake emit radiation at the plasma frequency. The back action of the radiation on the vortex motion is responsible for enhanced dissipation. A brief summary and conclusions are given in Sec. V.

\section{EQUATION OF MOTION}

The effective action in imaginary time for a vortex with vorticity +1 and coordinate $\mathbf{r}(\tau)$, added to the array, is given by ${ }^{10,7}$

$$
\begin{aligned}
\mathcal{S}_{\text {eff }}= & \frac{1}{2} \sum_{\alpha \beta} \int d \tau d \tau^{\prime} \dot{r}_{\alpha}(\tau) M_{\alpha \beta}\left[\mathbf{r}(\tau)-\mathbf{r}\left(\tau^{\prime}\right), \tau-\tau^{\prime}\right] \dot{r}_{\beta}\left(\tau^{\prime}\right) \\
& +i \hbar \sum_{i} q_{i} \sum_{\alpha} \int d \tau \nabla_{\alpha} \Theta\left[\mathbf{r}(\tau)-\mathbf{r}_{i}\right] \dot{r}_{\alpha}(\tau) \\
& -\int d \tau \mathbf{f} \cdot[\hat{z} \times \mathbf{r}(\tau)]
\end{aligned}
$$

Here the first term is the kinetic energy of the vortex, which arises from integration of the charge fluctuations; it is ex- 
pressed in terms of the vortex mass tensor $M[\mathbf{r}(\tau), \tau]$. The second term is the contribution of a static background of external charges [the islands are labeled by the index $i$ $\left.=\left(i_{x}, i_{y}\right)\right]$. Finally, the third term takes into account a uniform external current $\mathbf{I}$, with $\mathbf{f}=h \mathbf{I} / 2 e$. In the second term the phase configuration around a vortex, $\Theta_{i j}=\arctan \left[\left(y_{i}\right.\right.$ $\left.\left.-y_{j}\right) /\left(x_{i}-x_{j}\right)\right]$, has been defined. However, we do not include any offset charges in this work, and hence drop the second term in what follows.

The vortex mass tensor is given by

$$
\begin{aligned}
& M_{\alpha \beta} {\left[\mathbf{r}(\tau)-\mathbf{r}\left(\tau^{\prime}\right), \tau-\tau^{\prime}\right] } \\
& \quad=\sum_{i j} \nabla_{\alpha} \Theta\left[\mathbf{r}(\tau)-\mathbf{r}_{i}\right] \mathcal{Q}_{i j, \tau \tau^{\prime}} \nabla_{\beta} \Theta\left[\mathbf{r}_{j}-\mathbf{r}\left(\tau^{\prime}\right)\right],
\end{aligned}
$$

where the connected charge-charge correlation function in the Fourier space $\mathcal{Q}_{\mathbf{k}, \omega_{n}}$ is assumed to be of the same form as given by the SCHA approximation: ${ }^{6}$

$$
\mathcal{Q}_{\mathbf{k}, \omega_{n}}=\frac{\omega_{p}}{8 \alpha} \frac{k^{2}}{\omega_{k}^{2}+\omega_{n}^{2}}
$$

with $\omega_{k}^{2}=\omega_{p}^{2}\left(1+\xi^{2} k^{2}\right) ; \quad \omega_{p}=\sqrt{8 E_{C} E_{J}}$ is the Josephson plasma frequency. The dimensionless correlation length $\xi(\alpha)$ (see, e.g., Ref. 6) characterizes the stiffening of the spinwave spectrum due to quantum corrections. The above form for the charge-charge correlation function, Eq. (3), provides the correct small- $\alpha$ limit as calculated within the low- $\alpha$ expansion, and displays also the expected form for large values of $\alpha{ }^{7}$ The correlation length $\xi(\alpha)$ can be calculated within different approximations. We assume that it is zero for $\alpha$ $=0$, that is for the classical system, and an increasing function when $\alpha$ increases, diverging at the superconductorinsulator transition, i.e., for a critical value that we denote by $\alpha_{c}$.

We perform the continuation to real times of the action $\mathcal{S}_{\text {eff }}$ as described, e.g., in Ref. 9. The resulting action is a functional of $\mathbf{x}(t)=\mathbf{r}_{1}-\mathbf{r}_{2}$, and $\mathbf{r}(t)=\left(\mathbf{r}_{1}+\mathbf{r}_{2}\right) / 2$, where $\mathbf{r}_{1}(t)$ and $\mathbf{r}_{2}(t)$ are the coordinates defined on the two branches of the Keldysh contour, respectively. We then derive the semiclassical equation of motion. Because the saddle point occurs for $\mathbf{x}(t)=0$, we are ignoring the possibility of decoherence effects, which are included in the off-diagonal terms with $\mathbf{r}_{1} \neq \mathbf{r}_{2}$ (that is with $\mathbf{x} \neq 0$ ). In the equation of motion that follows, $\mathbf{r}(t)$ can be interpreted as the classical coordinate of the center of mass of the vortex. Then, the real-time action generating the equation of motion for the vortex is $(T=0)$

$$
\begin{aligned}
\mathcal{S}= & \frac{1}{2} \int d t \int d t^{\prime} \dot{r}^{\alpha}(t) \mathcal{G}_{\alpha \beta}^{R}\left[\mathbf{r}(t)-\mathbf{r}\left(t^{\prime}\right), t-t^{\prime}\right] \dot{r}^{\beta}\left(t^{\prime}\right) \\
& +\int d t \mathbf{f} \cdot[\hat{z} \times \mathbf{r}(t)],
\end{aligned}
$$

$$
\begin{aligned}
\mathcal{G}_{\alpha \beta}^{R}[\mathbf{r}(t), t]= & \int \frac{d^{2} \mathbf{k}}{k^{4}}(\hat{z} \times \mathbf{k})_{\alpha}(\hat{z} \times \mathbf{k})_{\beta} \\
& \times \int \frac{d \omega}{2 \pi} \mathcal{Q}_{\mathbf{k}, \omega}^{R} e^{-i \omega t+i \mathbf{k} \cdot \mathbf{r}(t)} .
\end{aligned}
$$

Furthermore, $\mathcal{Q}_{\mathbf{k}, \omega}^{R}$ is the retarded continuation of $\mathcal{Q}_{\mathbf{k}, \omega_{n}}$, and $\dot{\mathcal{Q}}_{\mathbf{k}, \omega}^{R}=-i \omega \mathcal{Q}_{\mathbf{k}, \omega}^{R}$. From Eq. (4), the equation of motion follows to be

$$
\begin{aligned}
(\mathbf{f} \times \hat{z})_{\alpha}= & \int_{-\infty}^{+\infty} d t^{\prime}\left\{\frac{d}{d t} \mathcal{G}_{\alpha \beta}^{R}\left[\mathbf{r}(t)-\mathbf{r}\left(t^{\prime}\right), t-t^{\prime}\right] \dot{r}^{\beta}\left(t^{\prime}\right)\right. \\
& \left.-\dot{r}^{\gamma}(t) \nabla_{\alpha} \mathcal{G}_{\gamma \beta}^{R}\left[\mathbf{r}(t)-\mathbf{r}\left(t^{\prime}\right), t-t^{\prime}\right] \dot{r}^{\beta}\left(t^{\prime}\right)\right\} .
\end{aligned}
$$

This equation simplifies for a vortex moving with constant velocity, orthogonal to the driving current, ${ }^{4,6}$ which we choose as $\mathbf{f}=\left(0, f_{0}\right)$. In particular, Eq. (6) reduces to $f_{0}$ $=\eta v[\mathbf{v}=(v, 0)]$, with the static "friction coefficient" $\eta$ given by

$$
\eta=\int d^{2} \mathbf{k} \frac{k_{y}^{2}}{k^{4}} \int d \omega \dot{\mathcal{Q}}_{\mathbf{k}, \omega}^{R} \delta\left(\omega+k_{x} v\right)
$$

This expression clearly describes the spin-wave damping of the vortex moving in the array. $\mathcal{Q}^{R}$ is the retarded continuation of the correlator defined in Eq. (3),

$$
\mathcal{Q}_{\mathbf{k}, \omega}^{R}=k^{2} g^{R}(\omega, k)=E_{J} \frac{k^{2}}{\omega_{k}^{2}-\left(\omega+i 0^{+}\right)^{2}} .
$$

Due to parity, only the imaginary part of $g^{R}$ survives the $k_{x}$ integration, which yields a real value for $\eta$ :

$$
\eta=\pi E_{J} \int d^{2} k \frac{k_{y}^{2}}{k^{2}} \omega_{k} \delta\left(k_{x}^{2} v^{2}-\omega_{k}^{2}\right)
$$

This quantity is nonvanishing only for velocities above a certain threshold, $v_{\text {th }}$. This threshold velocity ${ }^{6,7}$ depends on the cutoff chosen in the $k$ integration, as described in the next section: the sharp cutoff procedure yields $v_{\text {th }}$ $=\omega_{p} \sqrt{\xi^{2}+1 / 4 \pi} \equiv v_{\text {th }}^{\prime}$, while the soft cutoff gives $v_{\text {th }}$ $=\omega_{p} \xi$. For velocities smaller than $v_{\text {th }}$, ballistic motion is possible.

\section{FREQUENCY-DEPENDENT LINEAR RESPONSE}

Let us now assume that, while the vortex is moving with constant velocity $v$, it is subjected to a small harmonic external perturbation. The trajectory of the vortex can be written as $\mathbf{r}(t)=v t \hat{x}+\delta \mathbf{r}(t)$ where $\delta \mathbf{r}(t)$ is a slowly varying function of time. By linearizing in $\delta \mathbf{r}=(\delta x, \delta y)$ and transforming to Fourier space, the equation of motion, Eq. (6), becomes

where 


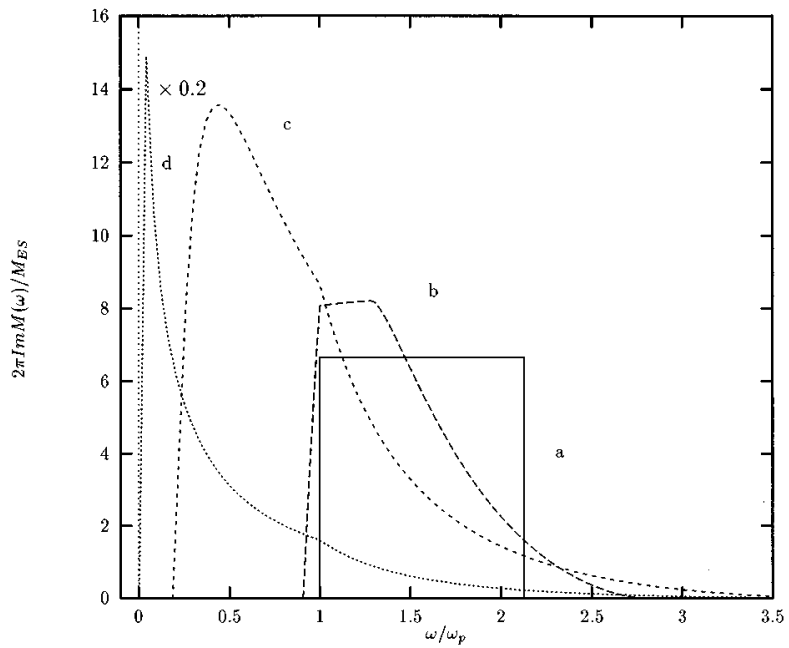

FIG. 1. $2 \pi \operatorname{Im} M(\omega) / M_{\mathrm{ES}}$ vs $\omega / \omega_{p}$ with sharp cutoff of the $k$ integration for $\xi=0.5$ and some values of the velocity below threshold, $v_{\text {th }}: \quad v / \xi \omega_{p}=0$ (a), 0.35 (b), 1.03 (c), 1.14 (d). The range of frequencies where $\operatorname{Im} \chi_{x x} \neq 0$ increases with increasing velocity. There is no damping $\left(\operatorname{Im} \chi_{x x}=0\right)$ below a threshold frequency $\omega_{\text {th }}$, which decreases with increasing $v$, so that $\omega_{\text {th }}\left(v=v_{\text {th }}\right)=0$.

$$
\left(\begin{array}{c}
f_{y}(\omega) \\
-f_{x}(\omega)
\end{array}\right)=\left(\begin{array}{cc}
\chi_{x x}^{-1}(\omega) & 0 \\
0 & \chi_{y y}^{-1}(\omega)
\end{array}\right)\left(\begin{array}{c}
\delta x(\omega) \\
\delta y(\omega)
\end{array}\right) .
$$

Explicitly,

$$
\begin{aligned}
\chi_{x x}^{-1}(\omega)= & -i \int d^{2} k \frac{k_{y}^{2}}{k^{2}}\left(\omega+k_{x} v\right) \dot{g}^{R}\left(\omega+k_{x} v, k\right) \\
& +i \int d^{2} k \frac{k_{y}^{2}}{k^{2}} k_{x} v \dot{g}^{R}\left(k_{x} v, k\right), \\
\chi_{y y}^{-1}(\omega)= & -i \int d^{2} k \frac{k_{x}}{k^{2}}\left(\omega k_{x}-v k_{y}^{2}\right) \dot{g}^{R}\left(\omega+k_{x} v, k\right) \\
& -i \int d^{2} k \frac{k_{y}^{2} k_{x} v}{k^{2}} \dot{g}^{R}\left(k_{x} v, k\right) \\
& +v \int d^{2} k\left(\omega k_{x}-v k_{y}^{2}\right) g^{R}\left(\omega+k_{x} v, k\right) \\
& +v^{2} \int d^{2} k k_{y}^{2} g^{R}\left(k_{x} v, k\right) .
\end{aligned}
$$

The kernel $g^{R}$ is defined in Eq. (8). Equation (10) shows that a Hall contribution does not appear within linear response, so that the $x$ and the $y$ component of the equation of motion can be considered separately. Therefore, we concentrate on the first equation, $f_{y}(\omega)=\chi_{x x}^{-1}(\omega) \delta x(\omega)$, with the following results:

$$
\operatorname{Re} \chi_{x x}^{-1}(\omega)=-E_{J}\left\{\int d^{2} k \frac{k_{y}^{2}}{k^{2}} \frac{\left(\omega+k_{x} v\right)^{2}}{\omega_{k}^{2}-\left(\omega+k_{x} v\right)^{2}}\right.
$$

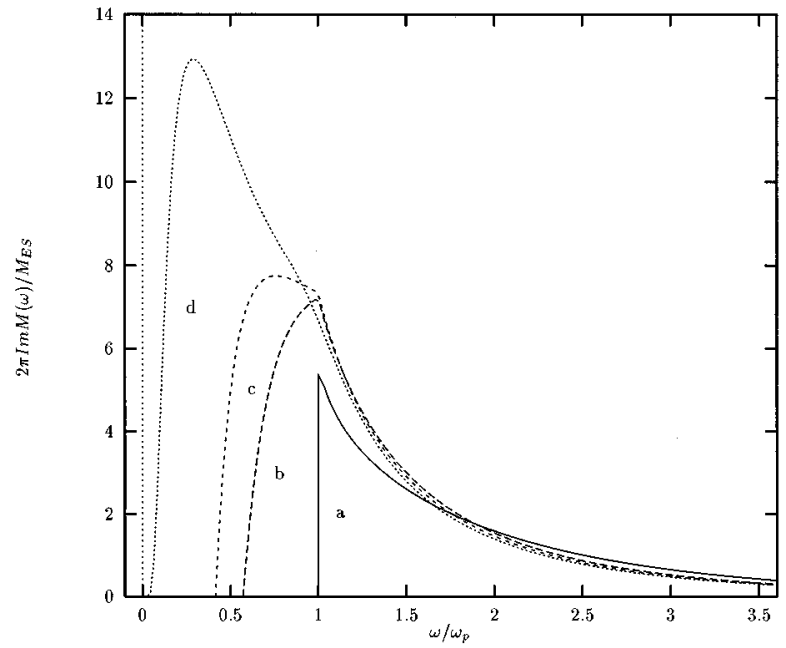

FIG. 2. $2 \pi \operatorname{Im} M(\omega) / M_{\mathrm{ES}}$ vs $\omega / \omega_{p}$ with soft cutoff of the $k$ integration for $\xi=0.5$ and some values of the velocity below threshold, $v_{\text {th }}=\xi \omega_{p}: v / \xi \omega_{p}=0$ (a), 0.8 (b), 0.9 (c), 0.99 (d).

$$
\begin{gathered}
\left.-\int d^{2} k \frac{k_{y}^{2}}{k^{2}} \frac{\left(k_{x} v\right)^{2}}{\omega_{k}^{2}-\left(k_{x} v\right)^{2}}\right\}, \\
\operatorname{Im} \chi_{x x}^{-1}(\omega)=-\pi E_{J} \int d^{2} k \frac{k_{y}^{2}}{k^{2}} \omega_{k}^{2} \delta\left(\left(\omega+k_{x} v\right)^{2}-\omega_{k}^{2}\right) .
\end{gathered}
$$

The zero-frequency limit of these equations shows that there is no real part of the response when the vortex is in steadystate motion, while the imaginary part yields the result given in Eq. (9). However, the zero frequency limit of $-\operatorname{Re} \chi_{x x}^{-1}(\omega) / \omega^{2}$ is related to the dynamical mass of the moving vortex and is nonvanishing.

\section{A. Imaginary part}

The imaginary part of the response describes the "dynamical spin-wave damping" of the vortex. When the frequency is nonzero, damping is present also below the threshold velocity, provided the frequency is higher than a threshold value $\omega_{\text {th }}$, which depends on velocity. This threshold frequency vanishes when the velocity approaches $v_{\text {th }}$. Performing a change of variables, $\left(k_{x}, k_{y}\right) \rightarrow\left(k_{x}, k\right)$, Eq. (14) becomes

$$
\begin{aligned}
\operatorname{Im} \chi_{x x}^{-1}(\omega)= & -2 \pi E_{J} \int d k \frac{\omega_{k}^{2}}{k} \int_{-k}^{k} d k_{x} \sqrt{k^{2}-k_{x}^{2}} \\
& \times \delta\left[\left(\omega+k_{x} v\right)^{2}-\omega_{k}^{2}\right]
\end{aligned}
$$

Clearly, because $\operatorname{Im} \chi_{x x}^{-1}(\omega)=-\operatorname{Im} \chi_{x x}^{-1}(-\omega)$, we may restrict ourselves to $\omega \geqslant 0$ in the following. Two different procedures are used to make the integral over $k$ convergent at large values of the wave vector. The integration can be cut sharply at $k=\bar{k}$, choosing $\bar{k}=\sqrt{4 \pi}$ in order to preserve the area of the first Brillouin zone. Alternatively, the integral is extended up to infinity but with an exponentially decreasing factor (soft cutoff, see, e.g., Ref. 4). From Eq. (15), we find for $v=0$ : 


$$
\operatorname{Im} \chi_{x x}^{-1}(\omega)= \begin{cases}-\frac{\pi^{2} E_{J} \omega^{2}}{2 \xi^{2} \omega_{p}^{2}} \vartheta\left(\omega-\omega_{p}\right)\left[1-\vartheta\left(\omega-\bar{\omega}_{p}\right)\right] & \text { sharp cutoff } \\ -\frac{\pi^{2} E_{J} \omega^{2}}{2 \xi^{2} \omega_{p}^{2}} \exp \left[-\sqrt{\left(\omega^{2}-\omega_{p}^{2}\right) / 2 \pi \omega_{p}^{2} \xi^{2}}\right] \vartheta\left(\omega-\omega_{p}\right) & \text { soft cutoff }\end{cases}
$$

where $\vartheta(x)$ is the unit step function and $\bar{\omega}_{p}=\sqrt{4 \pi} v_{\text {th }}^{\prime}$. Thus $\operatorname{Im} \chi_{x x}^{-1}(\omega)=0$ for $\omega<\omega_{\text {th }}$, where the threshold frequency $\omega_{\text {th }}$ coincides in the limit $v=0$ with the plasma frequency $\omega_{p}$. With increasing $v$, the range of frequencies where $\operatorname{Im} \chi_{x x}^{-1}(\omega) \neq 0$ increases with increasing velocity. No dynamical damping appears below $\omega_{\mathrm{th}}(v)$, which depends on velocity and vanishes for $v=v_{\text {th }}$. Explicitly, for the sharp cutoff,

$$
\omega_{\mathrm{th}}(v)= \begin{cases}0 & v \geqslant v_{\mathrm{th}}^{\prime} \\ \bar{\omega}_{p}-v \sqrt{4 \pi} & v_{\mathrm{th}}^{\prime} \geqslant v \geqslant \sqrt{4 \pi}\left(\omega_{p} \xi\right)^{2} / \bar{\omega}_{p} \\ \omega_{p} \sqrt{1-v^{2} /\left(\omega_{p} \xi\right)^{2}} & v \leqslant \sqrt{4 \pi}\left(\omega_{p} \xi\right)^{2} / \bar{\omega}_{p}\end{cases}
$$

while for the soft cutoff,

$$
\omega_{\text {th }}(v)=\left\{\begin{array}{ll}
0 & v \geqslant v_{\text {th }} \\
\sqrt{1-v^{2} /\left(v_{\text {th }}\right)^{2}} & v \leqslant v_{\text {th }}
\end{array} .\right.
$$

We plot $-\operatorname{Im} \chi_{x x}^{-1}(\omega) / \pi E_{J} \omega^{2}$ vs $\omega$, for a fixed value of $\xi=0.5$ and various velocities below threshold, in Figs. 1 and 2. The sharp cutoff procedure is adopted in Fig. 1, while the results with the soft cutoff are shown in Fig. 2. The quantity shown in this figure is directly related to the imaginary part of the frequency Fourier transform of the mass tensor, see Eq. (2), i.e., it equals $2 \pi \operatorname{Im} M(\omega) / M_{\mathrm{ES}}$.

\section{B. Real part}

The real part of the response, given by Eq. (13), is an even function of frequency; again $\omega \geqslant 0$ in the following. For $v=0$, we obtain

$$
\operatorname{Re} \chi_{x x}^{-1}(\omega)= \begin{cases}-\frac{\pi E_{J} \omega^{2}}{2 \xi^{2} \omega_{p}^{2}} \ln \left|\left(1+4 \pi \xi^{2}-\omega^{2} / \omega_{p}^{2}\right) /\left(1-\omega^{2} / \omega_{p}^{2}\right)\right| & \text { sharp cutoff } \\ -\frac{\pi E_{J} \omega^{2}}{\xi^{2} \omega_{p}^{2}} \int_{0}^{\infty} x d x e^{-x / \sqrt{2 \pi \xi^{2}}}\left[\left(1-\omega^{2} / \omega_{p}^{2}\right)+x^{2}\right]^{-1} & \text { soft cutoff }\end{cases}
$$

For $0<v<v_{\text {th }}\left(\right.$ or $\left.v_{\text {th }}^{\prime}\right)$, in a first step, we find

$$
\operatorname{Re} \chi_{x x}^{-1}(\omega)=\pi E_{J} \int d k \frac{\omega_{k}}{k v^{2}}\left\{\begin{array}{cc}
\sqrt{\left(\omega_{k}+\omega\right)^{2}-k^{2} v^{2}}-2 \sqrt{\omega_{k}^{2}-k^{2} v^{2}} & \text { if }\left|\omega_{k}-\omega\right|<k v<\omega_{k} \\
\sqrt{\left(\omega_{k}+\omega\right)^{2}-k^{2} v^{2}}-2 \sqrt{\omega_{k}^{2}-k^{2} v^{2}} & \\
+\operatorname{sign}\left(\omega_{k}-\omega\right) \sqrt{\left(\omega_{k}-\omega\right)^{2}-k^{2} v^{2}} & \text { if }\left|\omega_{k}-\omega\right|>k v .
\end{array}\right.
$$

In Figs 3 and 4 , we show $-\operatorname{Re} \chi_{x x}^{-1}(\omega) \omega_{p}^{2} / \pi E_{J} \omega^{2}$ $=2 \pi \operatorname{Re} M(\omega) / M_{\mathrm{ES}}$ vs $\omega / \omega_{p}$ (sharp cutoff: Fig. 3; soft cutoff: Fig. 4), for the same values of $\xi=0.5$ and $v$ below threshold as in Figs. 1 and 2. Note that $\lim _{\omega \rightarrow \infty} \operatorname{Re} \chi^{-1}(\omega)$ $=M_{\mathrm{ES}} \omega_{p}^{2}$. As is apparent from the figures, the real part has a logarithmic divergence (algebraic if $\xi=0$ ) when $v=0$, which disappears for finite $v$. The maximum of $2 \pi \operatorname{Re} M(\omega) / M_{\mathrm{ES}}$ increases in magnitude, and moves to lower frequencies, with increasing vortex velocity. In Fig. 5, we plot the same quantity as in Fig. 4, for a much larger value of $\xi, \xi=10$. Clearly, at small velocities, the response flattens over a large range of frequencies, and the maximum almost disappears. Going back to real time, this means that increasing the charge-charge correlation length implies a decrease of the response in time of the system. In the limit of low frequencies, Eq. (20) leads to

$$
\operatorname{Re} \chi_{x x}^{-1}(\omega \simeq 0)=-\omega^{2} \pi E_{J} \int d k \frac{k \omega_{k}}{\left(\omega_{k}^{2}-k^{2} v^{2}\right)^{3 / 2}}+O\left(\omega^{4}\right)
$$

In Fig. 6 we plot the zero-frequency limit as the ratio $M_{\mathrm{dyn}} / M_{\mathrm{ES}}$ vs velocity, where we defined $M_{\mathrm{dyn}}=M(\omega=0)$ (sharp cutoff). In particular, $M_{\text {dyn }}$ diverges for $v \rightarrow v_{\text {th }}$. This divergence corresponds to the vanishing of the acceleration of the vortex while entering the diffusive regime.

\section{RADIATIVE DISSIPATION OF A QUANTUM VORTEX}

In the previous section, we have studied the linear response of a vortex to an external sinusoidal perturbation, while it is moving with constant velocity. There is one case, however, in which linear response is unsatisfactory, namely, when the spin wave emitted by the vortex is reabsorbed 


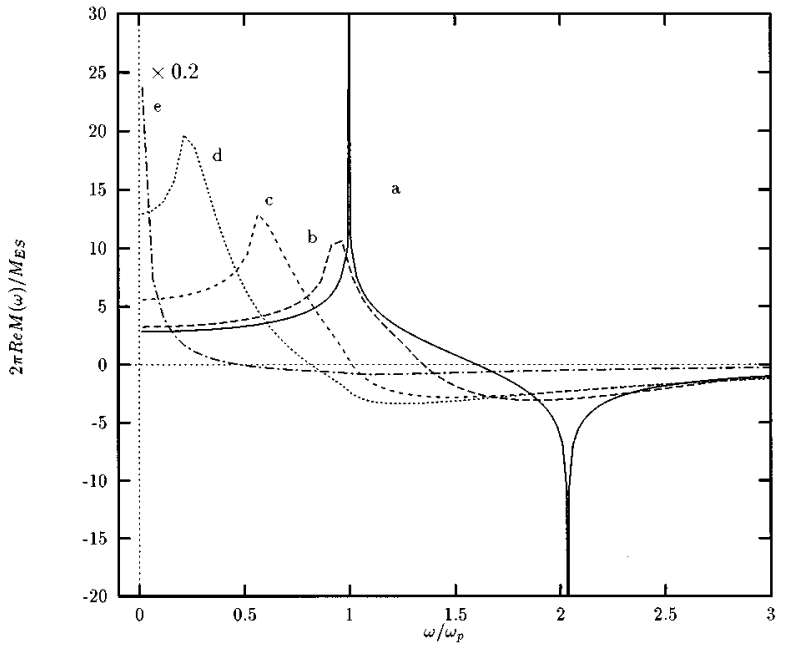

FIG. 3. $2 \pi \operatorname{Re} M(\omega) / M_{\mathrm{ES}}$ vs $\omega / \omega_{p}$ with sharp cutoff of the $k$ integration for $\xi=0.5$ and some values of the velocity below threshold: $\quad v / \xi \omega_{p}=0$ (a), 0.35 (b), 0.8 (c), 1.03 (d), 1.14 (e). The divergences at zero velocity disappear when $v \neq 0$.

again, so that the nonlinearity of the back action cannot be neglected. To understand how this happens physically, let us consider the classical case, so that the dispersion of the spinwave spectrum can be ignored. The spectrum then consists of just one single-frequency $\omega_{p}$ that is a localized mode for each separate junction. When the vortex passes a junction, the phase difference at the junction left behind changes by a value of order $\pi$, and according to the Josephson relation a voltage pulse of amplitude $V_{0} \approx \pi \hbar v / 2 e$ arises. If we denote the response of the junction to this pulse by $V_{1}(t)$ $=V_{1} \sin \omega_{p} t$, we expect a phase difference at the junction left behind that is roughly $\varphi(t)=\varphi_{0}+v \pi t / a$ $+\left(2 e V_{1} / \hbar \omega_{p}\right) \cos \omega_{p} t$. This implies a perturbation of the

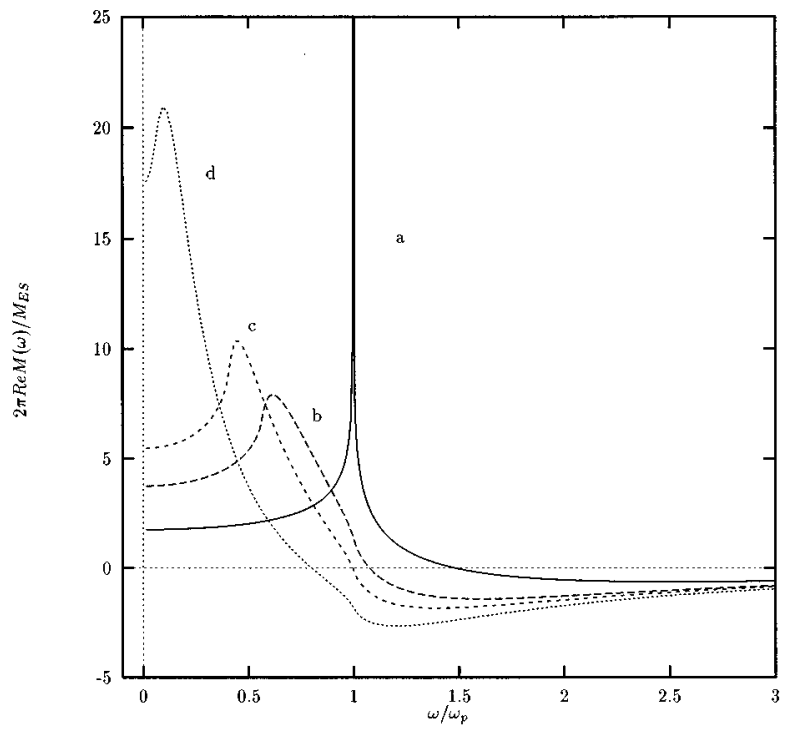

FIG. 4. $2 \pi \operatorname{Re} M(\omega) / M_{\mathrm{ES}}$ vs $\omega / \omega_{p}$ with soft cutoff of the $k$ integration for $\xi=0.5$ and some values of the velocity below threshold: $\quad v / \xi \omega_{p}=0$ (a), 0.8 (b), 0.9 (c), 0.99 (d).

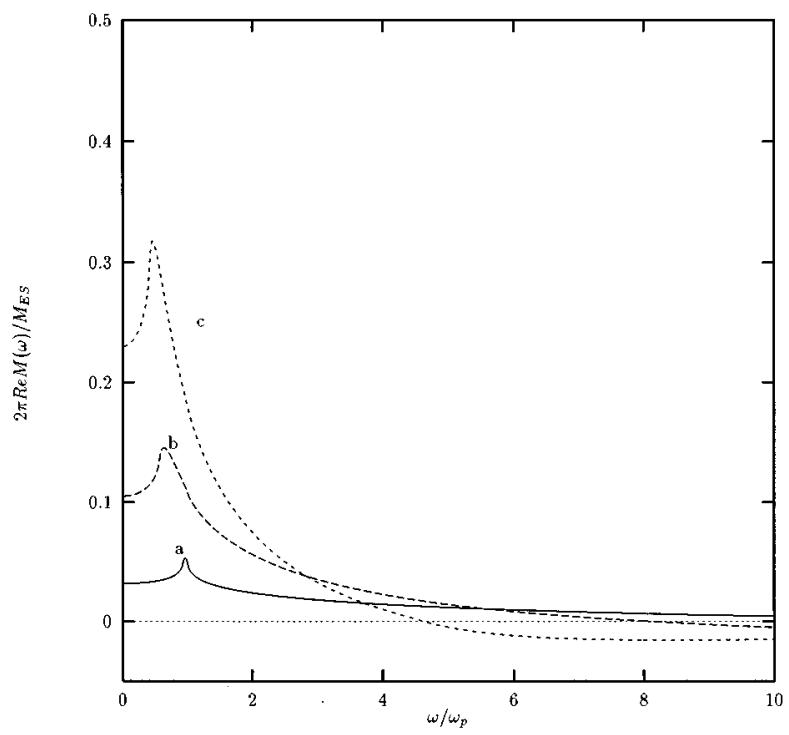

FIG. 5. $2 \pi \operatorname{Re} M(\omega) / M_{\mathrm{ES}}$ vs $\omega / \omega_{p}$ with soft cutoff of the $k$ integration for $\xi=10$ and some values of the velocity below threshold: $\quad v / \xi \omega_{p}=0.3$ (a), 0.8 (b), 0.9 (c).

vortex trajectory, which will be of the form $x(t)=x_{0}+v t$ $+\gamma \cos \omega_{p} t$. We have defined $\gamma=\left(2 e a V_{1} / \pi \hbar \omega_{p}\right)$ as the strength of the coupling between radiation and vortex. Because no power can be extracted from the moving vortex, the driving force times the velocity has to be positive at any time, which implies $\gamma<v / \omega_{p}$. This mechanism of radiative dissipation for a moving vortex was considered already in Ref. 11 for the classical case. Its contribution, $\eta_{p}$, to the viscosity of the vortex motion in strongly underdamped arrays (McCumber parameter $\beta_{c}=2 \pi I_{c} R_{N}^{2} C / \phi_{0} \gg 1$ ) was estimated to be much larger than $\eta_{\mathrm{BS}}$, the viscosity in the Bardeen-Stephen model $\left(\eta_{p}=\eta_{\mathrm{BS}} \sqrt{\beta_{c}} / 2 \pi\right)$.

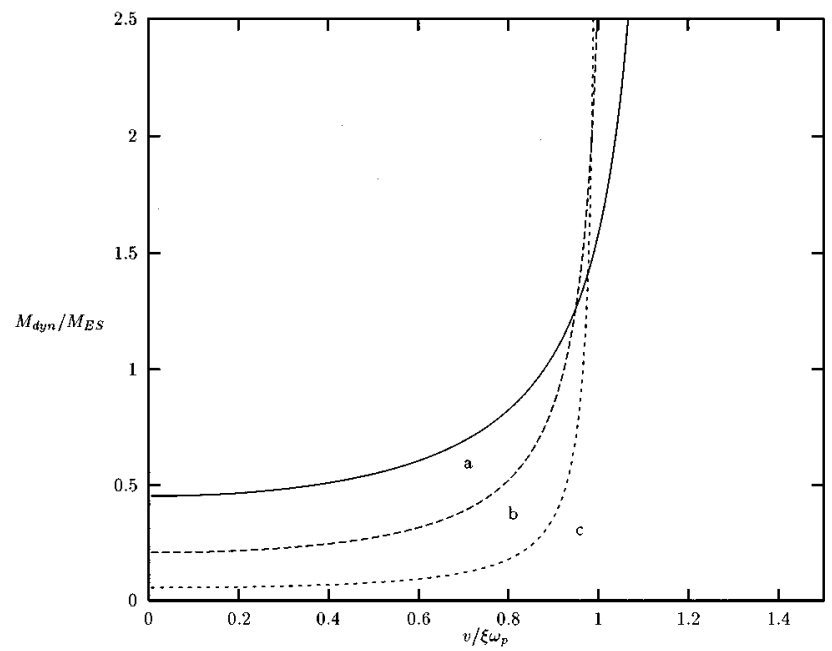

FIG. 6. Normalized dynamical mass $M_{\mathrm{dyn}} / M_{\mathrm{ES}}\left[M_{\mathrm{dyn}}=M(\omega\right.$ $=0)]$ vs $v / \xi \omega_{p}$, for $\xi=0.5$ (a), 1.0 (b), 2.5 (c) (sharp cutoff of the $k$ integration). 


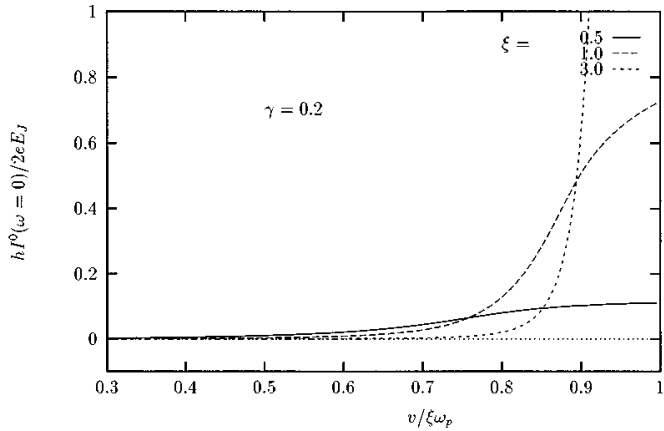

FIG. 7. Zero-frequency component of the external current $h I^{0} / 2 e E_{J}$ vs velocity $v / \xi \omega_{p}$ below threshold, for $\xi=0.5,1.0,3.0$; $\gamma=0.2$.

In the quantum case, using Eq. (6) and assuming again that the motion is orthogonal to the driving force, we have

$$
\begin{aligned}
f(t)= & \int d^{2} k \frac{k_{y}^{2}}{k^{4}} \int \frac{d \omega}{2 \pi}(-i \omega) \mathcal{Q}_{\mathbf{k}, \omega}^{R} \cdot \int_{-\infty}^{\infty} d t^{\prime} \\
& \times \dot{x}\left(t^{\prime}\right) e^{i \omega\left(t^{\prime}-t\right)+i k_{x}\left[x\left(t^{\prime}\right)-x(t)\right]} .
\end{aligned}
$$

Fourier transforming both with respect to $t$ and to $t^{\prime}$, we arrive at

$$
\begin{aligned}
f(\omega)= & 2 \pi i \sum_{n, m} \delta\left(\omega-m \omega_{p}\right) \int d^{2} k \frac{k_{y}^{2}}{k_{x} k^{4}} \\
& \times\left[(n+m) \omega_{p}-k_{x} v\right]^{2} \mathcal{Q}_{\mathbf{k},(n+m) \omega_{p}-k_{x} v}^{R} \\
& \times i^{m} J_{n}\left(k_{x} \gamma\right) J_{m+n}\left(k_{x} \gamma\right) .
\end{aligned}
$$

For $\gamma$ equal to zero, only the term with $n=m=0$ survives, corresponding to the constant-velocity result of Eq. (7). Using that the real part of $\mathcal{Q}^{R}$ is even in $\omega$, while the imaginary part is odd, we rewrite the constant current contribution, given by the $m=0$ term, as

$$
\begin{aligned}
f(0)= & -2 \pi \sum_{n \geqslant 0}\left(2-\delta_{n, 0}\right) \int d^{2} k \frac{k_{y}^{2}}{k^{4} k_{x}}\left(n \omega_{p}-k_{x} v\right)^{2} J_{n}^{2}\left(k_{x} \gamma\right) \\
& \times \operatorname{Im} \mathcal{Q}_{\mathbf{k}}^{R}\left(n \omega_{p}-k_{x} v\right) .
\end{aligned}
$$

We denote by $f_{0 n}$ one of the terms of this sum. Inserting the imaginary part of $\mathcal{Q}^{R}$ as obtained from Eq. (8), we obtain

$$
\begin{aligned}
f_{0 n}= & -(2 \pi)^{2} E_{J}\left(2-\delta_{0, n}\right) \int_{0}^{\infty} d k e^{-k / k_{c}} \frac{\omega_{k}^{2}}{k} \int_{-k}^{k} \frac{d k_{x}}{k_{x}} \\
& \times \sqrt{k^{2}-k_{x}^{2}} J_{n}^{2}\left(k_{x} \gamma\right) \delta\left[\left(n \omega_{p}-k_{x} v\right)^{2}-\omega_{k}^{2}\right]
\end{aligned}
$$

The integral over $k_{x}$ is performed straightforwardly, while the integral over $k$ is turned into an integral over the energy of the spin-wave modes. The sum over integers converges rapidly ( $n=5$ is sufficient), because the coupling $\gamma$ is assumed to be small. Under this change of variables, the range of frequencies to be integrated over is restricted by the $\delta$ functions, and further by the cutoff to values between $\omega_{p}$ and $\bar{\omega}_{p}$.

The average component of the current is plotted in Fig. 7 vs the velocity of the vortex, for various values of $\xi$, keeping

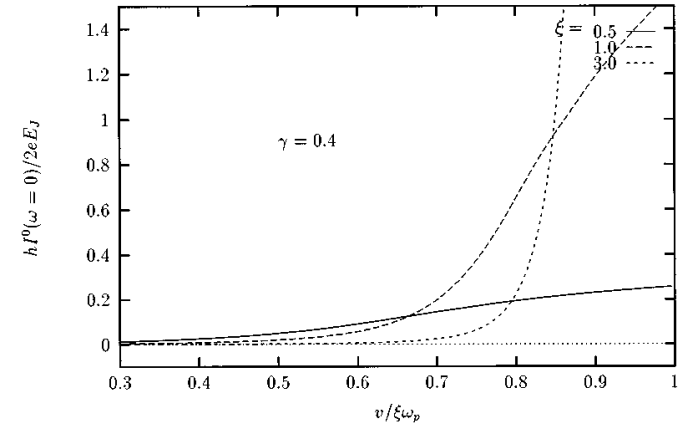

FIG. 8. Zero-frequency component of the external current $h I^{0} / 2 e E_{J}$ vs velocity $v / \xi \omega_{p}$ below threshold, for $\xi=0.5,1.0,3.0$; $\gamma=0.4$.

the coupling $\gamma$ fixed. There is no sharp velocity threshold for diffusive motion, especially at low $\xi$. A stationary driving force is required during the radiation process to sustain the drift motion. This constant current has to increase steadily with increasing $v$. Therefore, ballistic motion becomes fragile when $\xi$ is small. For larger $\xi$ values, the threshold between ballistic and diffusive motion remains sharp. However, it is shifted to lower velocities when the coupling $\gamma$ increases (see Fig. 8).

It can be easily understood why the ballistic window is robust only for large $\xi$ values, i.e., high quantumness of the vortex. For small $\xi$, the spin-wave excitation spectrum has little dispersion. According to a Galilei's transformation, to excite spin waves, the $k$ components of the moving vortex have to satisfy the inequality $\omega_{k}-k_{x} v<0$. When $\xi$ is small, spin waves can be excited, provided $v>\omega_{p} / \sqrt{4 \pi}$, and the dissipation increases steadily with velocity, but remains rather low. On the contrary, when $\xi$ increases, the most substantial contribution, coming from small $k$ vectors, require large velocities to be excited. Instead, a large maximum of dissipation is developed, close above the threshold velocity $v_{\text {th }}$, as soon as spin waves are available for excitation (see Fig. 9).

\section{SUMMARY AND CONCLUSION}

The real-time equation of motion of a quantum vortex moving in a Josephson junction array has been investigated for any $\alpha=\sqrt{E_{C} / 8 E_{J}}$, up to the superconductor-insulator

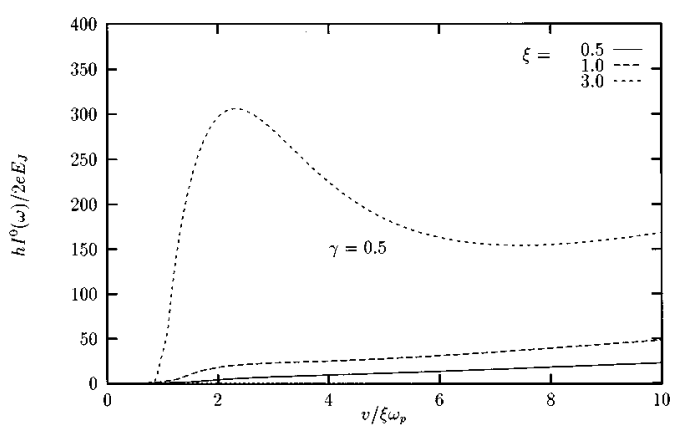

FIG. 9. Zero-frequency component of the external current $h I^{0} / 2 e E_{J}$ vs velocity $v / \xi \omega_{p}$ above the threshold velocity, for $\xi$ $=0.5,1.0,3.0 ; \gamma=0.5$. 
phase transition. ${ }^{3}$ By increasing $\alpha$, the correlation length $\xi$ increases, and quantum effects become more and more important; $\xi$ diverges at the transition. The equation of motion is nonlocal in time, clearly not of the form of Newton's law (even when a damping force is added). The frequencydependent response to an external driving current has been analyzed in the linear response regime. At small frequencies, quantum effects produce a rather flat response, that is the response becomes more and more instantaneous. In fact, the kernel oscillates with time, and shows a first peak at a time which is roughly the delay time for the system. By increasing $\xi$, the peak becomes sharper, and the delay decreases. Our interpretation is that at higher $\xi$ values, the vortex behaves more like a quantum particle. Both the delay time and the width go to zero when $\xi \rightarrow \infty$. In this limit a phenomenological Newton's law, $f=M_{\mathrm{dyn}} \dot{v}$, for the vortex is recovered, which defines the dynamical mass, $M_{\text {dyn }}$. This mass differs from the thermodynamical mass, $M_{v}$, introduced previously. ${ }^{6,7}$ The latter is not to be expected to govern the quantum dynamics; it only coincides with $M_{\text {dyn }}$, independent of the approximation used to evaluate it, when the vortex experiences the perturbing force while being initially at rest. On the other hand, $M_{\text {dyn }}$ is velocity-dependent and increases with the initial velocity (see Fig. 6). It is found to be larger than $M_{\mathrm{ES}}$ close to the threshold velocity $v_{\text {th }}$ only, at which purely diffusive motion sets in. This result is qualitatively in agreement with what follows from recent experiments on vortex dynamics, ${ }^{12}$ which estimate larger vortex masses than those calculated theoretically up to now.

Beyond linear response, we also considered an oscillating trajectory for the vortex at frequency $\omega_{p}$. This describes emission of radiation by the junctions in the wake of a moving vortex, which acts back onto the vortex itself. The zerofrequency component of the current gives information about the resulting additional dissipation. The ballistic window becomes fragile, particularly for small $\xi$. In fact, dissipation is present for small $\xi$ almost over the whole range of velocities, and the sharp threshold between ballistic and diffusive motion of the vortex is lost. A rather clear crossover between the two regimes is found when $\xi$ is large, and the coupling of the vortex to the driving field is small.

We point out that because a single vortex is considered here, Eq. (22) is not periodic in the vortex trajectory (in contrast to the Josephson current in a single junction, where the phase difference is the corresponding variable). This implies that there is no lock-in between $V_{0}$ and the frequency of radiation, and therefore no Shapiro steps (which appear when a single junction is exposed to microwave radiation). The absence of any sharp features in the vortex characteristics is related to the fact that quantum fluctuations imply a retardation between current and voltage, and the integration over frequencies smears out any sharp matching.

\section{ACKNOWLEDGMENTS}

We thank G. Falci, R. Fazio, J. Kissner, and A. van Otterlo for discussions. The authors enjoyed the hospitality of the Institute of Scientific Interchange (ISI), Torino, where part of this work was performed. Financial support by CNR (Italy) (Contract No. 115.22594) is also acknowledged.
${ }^{1}$ U. Eckern and A. Schmid, Phys. Rev. B 39, 6441 (1989).

${ }^{2}$ B. J. van Wees, Phys. Rev. Lett. 65, 255 (1990).

${ }^{3}$ R. Fazio and G. Schön, Phys. Rev. B 43, 5307 (1991).

${ }^{4}$ U. Eckern and E. B. Sonin, Phys. Rev. B 47, 505 (1993).

${ }^{5}$ U. Geigenmüller, C. J. Lobb, and C. B. Whan, Phys. Rev. B 47, 348 (1993).

${ }^{6}$ R. Fazio, A. van Otterlo, and G. Schön, Europhys. Lett. 25, 453 (1994).

${ }^{7}$ G. Luciano, U. Eckern, and J. G. Kissner, Europhys. Lett. 32, 669 (1995); G. Luciano, U. Eckern, J. G. Kissner, and A. Tagliacozzo, J. Phys.: Condens. Matter 8, 1241 (1996).

${ }^{8}$ H. S. J. van der Zant, F. C. Fritschy, and J. E. Mooij, Europhys. Lett. 19, 541 (1992); H. S. J. van der Zant, F. C. Fritschy, W. J.
Elion, L. J. Geerligs, and J. E. Mooij, Phys. Rev. Lett. 69, 2971 (1992).

${ }^{9}$ U. Eckern, G. Schön, and V. Ambegaokar, Phys. Rev. B 30, 6419 (1984).

${ }^{10}$ J. E. Mooij and G. Schön, in Single Charge Tunnelling, Vol. 294 of NATO Advanced Study Institute, Series B: Physics, edited by H. Grabert and M. H. Devoret (Plenum, New York, 1992), p. 275.

${ }^{11}$ P. A. Bobbert, Phys. Rev. B 45, 7540 (1992).

${ }^{12}$ H. S. J. van der Zant, F. C. Fritschy, T. P. Orland, and J. E. Mooij, Europhys. Lett. 18, 343 (1992); Phys. Rev. B 47, 295 (1993). 